\title{
Radial Basis Functional Model of Multi-Point Dieless Forming Process for Springback Reduction and Compensation
}

\author{
Misganaw Abebe ${ }^{(D)}$, Jun-Seok Yoon and Beom-Soo Kang * \\ Department of Aerospace Engineering, Pusan National University, Busan 46241, Korea; \\ misge98@gmail.com (M.A.); hamjang21c@gmail.com (J.-S.Y.) \\ * Correspondence: bskang@pusan.ac.kr; Tel.: +82-51-510-2310; Fax: +82-51-512-4491
}

Received: 29 September 2017; Accepted: 23 November 2017; Published: 27 November 2017

\begin{abstract}
Springback in multi-point dieless forming (MDF) is a common problem because of the small deformation and blank holder free boundary condition. Numerical simulations are widely used in sheet metal forming to predict the springback. However, the computational time in using the numerical tools is time costly to find the optimal process parameters value. This study proposes radial basis function (RBF) to replace the numerical simulation model by using statistical analyses that are based on a design of experiment (DOE). Punch holding time, blank thickness, and curvature radius are chosen as effective process parameters for determining the springback. The Latin hypercube DOE method facilitates statistical analyses and the extraction of a prediction model in the experimental process parameter domain. Finite element (FE) simulation model is conducted in the ABAQUS commercial software to generate the springback responses of the training and testing samples. The genetic algorithm is applied to find the optimal value for reducing and compensating the induced springback for the different blank thicknesses using the developed RBF prediction model. Finally, the RBF numerical result is verified by comparing with the FE simulation result of the optimal process parameters and both results show that the springback is almost negligible from the target shape.
\end{abstract}

Keywords: multi-point dieless forming; springback reduction; springback compensation; radial basis function

\section{Introduction}

Springback is a common and critical existence in sheet metal forming processes, which is caused by the elastic redistribution of the internal stresses after unloading of the external forces. Since multi-point dieless forming (MDF) is only a multi-curvature bending and small deformation, the tendency of inducing springback is high. Springback prediction using finite element (FE) simulation is familiar for any types of forming processes.

Previous researchers are investigated and proposed some techniques to compensate and reduce springback on MDF using numerical simulation and physical experiment, such as, Li et al. [1] proposed the multi-step forming to reduce the unloading springback on multi-point forming using numerical simulation. Hwang et al. [2] introduced the springback adjustment for multi-point forming of thick plate in shipbuilding using FE analysis and the iterative displacement adjustment algorithm. Shim et al. [3] investigates the tension force on MDF stretch forming process of aluminum alloy sheet to reduce the induced springback using FE simulation. However, using only FE simulation to compensate the machine tool geometry or any design or process parameters is computationally expensive. To overcome this problem, this study proposed surrogate modeling to replace the computational expensive FE simulation as a function of target curvature radius, blank thickness, and punch holding time (stress relief period) after the final displacement of the punch. 
The application of surrogate modeling on sheet metal forming were investigated by different researchers, for instance, Liu and Liang [4] proposed a fuzzy genetic algorithm to compensate for springback of multi-curvature forming. Zhang et al. [5] introduced an algorithm to compensate springback by modifying the double curved plate. Kitayama and Yoshioka [6] proposed a springback reduction technique with the control of punch speed and blank holder force through sequential approximate optimization using the radial basis function (RBF) network. Behera et al. [7] proposed a solution to improve the accuracy of single point incremental sheet forming by using multivariate adaptive regression splines as an error prediction tool to generate continuous error response surfaces for individual features and feature combinations. In order to reduce springback effects after forming, $\mathrm{Li}$ [8] also deals with the variable of blank holder force using the least square support vector regression by establishing the adaptive metamodeling optimization system. Khada and El-Morsy [9] applied Kriging metamodel to predict the springback in the air bending process with process parameters of blank material and geometry. Hassen et al. [10] investigates the springback for deep drawing process using the parameters of blank holding force and friction coefficient as a function of time. In this study, the sensitivity analyses of the finite element simulation result were investigated by applying a statistical method. The influence of the parameter variation also analyzed using a sequential screening experiment by applying a piecewise constant function. Karaağaç [11] carried experimental investigation to evaluate the process parameters of bending pressure, bending die angle, punch holding time, and the rubber membrane thickness effect on springback in V-bending using the flexforming process, and he also used the fuzzy logic system to estimate the springback effect. In [12,13] also investigated the influence of punch holding time and other process parameters for V-bending dies. In addition, different researchers [14-16] have applied the surrogate model to investigate springback with a consideration of different process parameters. However, these techniques were applied to single die forming, and most of the study used blank holder to reduce the induced springback; so this study investigates springback for blank holder free boundary condition of the MDF process to fill the gap.

This study proposes process optimization along with surrogate modeling with the aim of reducing and compensating springback in the forming of AA3003-H14 aluminum alloy sheet of saddle shape by MDF with consideration of three influencing parameters: curvature radius, blank thickness, and punch holding time. To construct the model, first, the study chose Latin hyper cube design of experiment (DOE) method for training and random sampling for testing. The Latin hypercube DOE method facilitates the statistical analyses and the extraction of a prediction model. FE simulation is applied using ABAQUS commercial software (ABAQUS 6.12, Dassault Systèmes Simulia Corp., Providence, RI, USA) to generate the training and testing sample data responses. After obtaining the training sample responses from the numerical simulation, different prediction model has been applied such as regression model, Ordinary and Blind Kriging, and RBF. The $R^{2}$ and roots mean square error (RMSE) numerical validation of the testing data, which has the same number of the training sample shown $\mathrm{RBF}$ is more acceptable than the others. Then, the study used the prediction model to apply the genetic algorithm (GA) in MATLAB to determine the global optimal parameter's value. First, the study found the optimal value of punch holding time to reduce the spring back for different blank thickness. Second, the proper curvature radius value is determined to compensate the induced springback for $800 \mathrm{~mm}$ target curvature radius at a different blank thickness. Finally, the FE simulation result of the optimization process parameters shows that the springback is almost negligible from the target geometry.

The rest of the paper is organized as follows. Section 2 discusses the data acquisition methods, using FE methods it includes the geometry, material, and numerical simulation model, in addition, the defect quantification method is also discussed. Section 3 describes the process parameters, sampling technique, formulation of the RBF, and finally the numerical model verification is discussed to validate the developed prediction model. Section 4 presents the optimal process parameter result to compensate the induced springback for the given target shape. 


\section{Finite Element Modeling}

\subsection{Geometry Model}

The study investigates the MDF springback effect on the rectangular saddle shape, which is shown in Figure 1a. The general equation of the saddle geometry is obtained from: $Z(x, y)=\sqrt{R_{y}^{2}-y^{2}}-\sqrt{R_{x}^{2}-x^{2}}$ where $x, y$ and $z$ are the Cartesian coordinates, $R_{x}$ is the radius curvature along the $x$-axis and $R_{y}$ is the radius curvature along the $y$-axis.

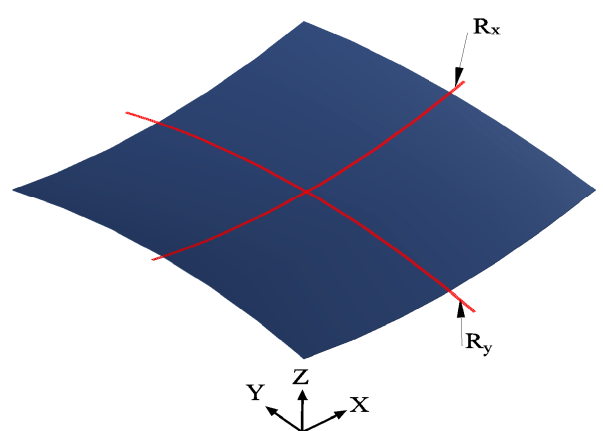

(a)

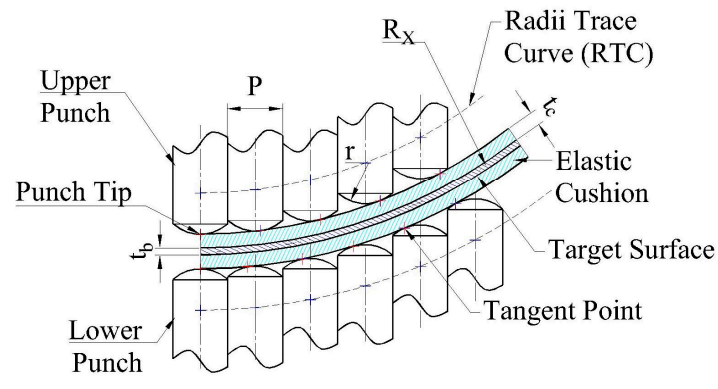

(b)

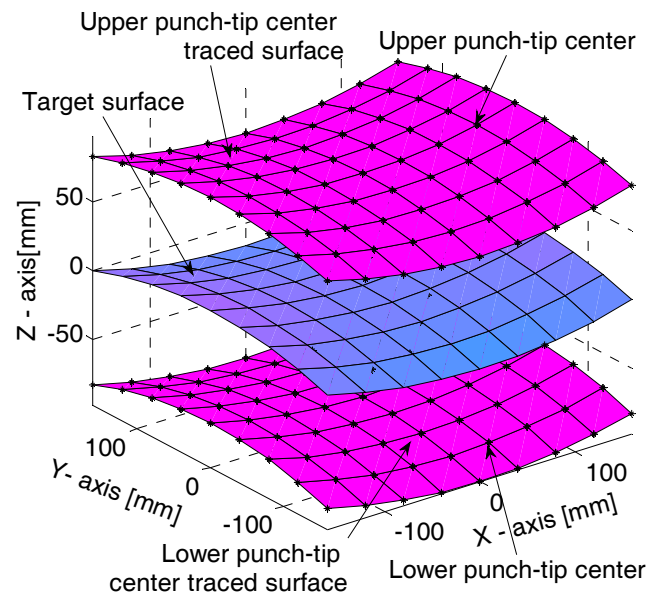

(c)

Figure 1. Saddle shape geometry model on multi-point dieless forming (MDF): (a) Target shape; (b) Punch placement in a two-dimensional view; (c) Positions of the lower and upper Radii trace curve (RTC) radii center traced surfaces.

The MDF target surface is generated using the discrete punches element, which has the $m$ number of punches along the $x$-axis and $n$ number of punches along the $y$-axis. To construct the target saddle shape in MDF, the lower and the upper punch matrices are placed using Equations (1) and (2), respectively [9].

$$
\begin{aligned}
& Z_{\mathrm{L}}\left(x_{i j}, y_{i j}\right)=\sqrt{\left(R_{y}^{\mathrm{L}}\right)^{2}-y_{i j}^{2}}-\sqrt{\left(R_{x}^{\mathrm{L}}\right)^{2}-x_{i j}^{2}} \\
& Z_{\mathrm{U}}\left(x_{i j}, y_{i j}\right)=\sqrt{\left(R_{y}^{\mathrm{U}}\right)^{2}-y_{i j}^{2}}-\sqrt{\left(R_{x}^{\mathrm{U}}\right)^{2}-x_{i j}^{2}}
\end{aligned}
$$

where $0<i<m ; 0<j<n ; m$ and $n$ are the number of punches along the $x$ - and $y$-axes, respectively; $R_{x}^{\mathrm{L}}$ and $R_{y}^{\mathrm{L}}$ are the curvature radii of the lower punch-tip radius center traced surface along the $x$ - and $y$-axes, respectively; $R_{x}^{\mathrm{U}}$ and $R_{y}^{\mathrm{U}}$ are the curvature radii of the upper punch-tip radius center traced surface along the $x$ - and $y$-axes, respectively. Figure $1 \mathrm{~b}$ shows the punch placement in a 2-dimensional 
view and Figure 1c shows the positions of the lower and upper RTC radii center traced surfaces, which is obtained using Equations (1) and (2), respectively.

\subsection{Material Model}

AA3003-H14 aluminum alloy sheet metal blank is used to investigate the springback effect on MDF. The material properties are taken from our previous study [17], which is also mentioned in Table 1. Since the MDF process is a small deformation, the study treated the sheet material as an isotropic property, in addition, here, the MDF process is not subjected to cyclic loading so that the study chose isotropic hardening instead of kinematic hardening. Swift strain hardening model is adopted for the material modeling which is $\sigma=K\left(\varepsilon_{0}+\varepsilon\right)^{n}$ for $\sigma \geq \sigma_{y}$, where $K$ is the strength coefficient, $\varepsilon$ is the true strain value, $\varepsilon_{0}$ is the pre-strain constant, and $n$ is the strain hardening exponent.

Table 1. Material properties of AA3003-H14.

\begin{tabular}{ccc}
\hline Properties & Value & Unit \\
\hline Young's Modulus $(E)$ & 70.1 & $\mathrm{GPa}$ \\
Poison ratio $(v)$ & 0.33 & $\mathrm{~N} / \mathrm{A}$ \\
Density $(\rho)$ & 2700 & $\mathrm{~kg} / \mathrm{m}^{2}$ \\
Yield stress $\left(\sigma_{y}\right)$ & 152.2 & $\mathrm{MPa}$ \\
Strength Coefficient $(K)$ & 192.7 & $\mathrm{MPa}$ \\
Strain hardening exponent $(n)$ & 0.0394 & $\mathrm{~N} / \mathrm{A}$ \\
Pre-strain constant $\left(\varepsilon_{0}\right)$ & $2.51 \times 10^{-3}$ & $\mathrm{~N} / \mathrm{A}$ \\
\hline
\end{tabular}

For elastic cushion, polyurethane material with a hardness of shore $90 \mathrm{~A}$ is chosen. Mooney-Rivlin hyperplastic modeling is employed for the simulation model, the model equation is given as:

$$
U=C_{10}\left(\bar{I}_{1}-3\right)+C_{01}\left(\bar{I}_{2}-3\right),
$$

where $U$ is the strain energy per unit of initial volume, $\bar{I}_{1}$ and $\bar{I}_{2}$ are the first and the second deviatoric strain invariants, respectively, and $C_{10}$ and $C_{01}$ are the material constants. The martial constants are taken from the previous study [18], which are $C_{01}=0.6606$ and $C_{10}=-0.0057$.

\subsection{Simulation Model}

Both explicit and implicit schemes have been used in ABAQUS commercial software to solve a challenging MDF sheet metal forming process that involves a high amount of springback. First, the explicit FE formulation was used to solve the MDF loading process, because the model is highly discontinuous process due to the geometry complexity, material property, and discrete punch element, so the quadratic convergence may be lost, and it may require a larger time increment than the explicit method and several iterations to obtain a solution within the prescribed tolerances.

While the loading process is conducted, a deformable blank sheet was brought into contact with a pair of deformable elastic cushions and the cushion also in contact with the rigid upper and lower discrete punch elements. The contact friction coefficient between the blank and elastic cushion, and elastic cushion and punch is considered as 0.1 [19]. Blank sheet and elastic cushions are meshed using C3D8R solid element and R3D4 shell element is also used for the rigid punch element. To reduce the computational time, as shown in Figure 2, the symmetrical condition is considered. For $x$-symmetry, the translational displacement in the $x$-direction, and rotational in $y$-direction and $z$-direction were set to zero $\left(U_{x}=U R_{y}=U R_{z}=0\right)$, and under $y$-symmetry, translational displacement in $y$-direction, and rotation in $x$-direction and $z$-direction were set to zero $\left(U_{y}=U R_{x}=U R_{z}=0\right)$. After the completion of the loading process using explicit analysis, the deformed shape, strains, and stresses within the blank elements were transferred into the implicit analysis for unloading process. This was accomplished by creating a database file that updated the geometry and stress-strain history of the implicit elements 
to match the final explicit solution. During the implicit analysis, the punches and elastic cushions were removed from the model and valid boundary conditions were employed to the sheet blank to restrain rigid body motion. As shown in Figure 3, the displacement in $y$-axis (U2) at the center of the nodes are fixed, and the X-symmetric and Z-symmetric are also imposed, as shown in the figure. Finally, the implicit analysis was exploited to determine the succeeding springback displacement in the deformed blank that occurred after the forming load was removed. The FE simulation model was experimentally validated in our previous study $[17,18]$.

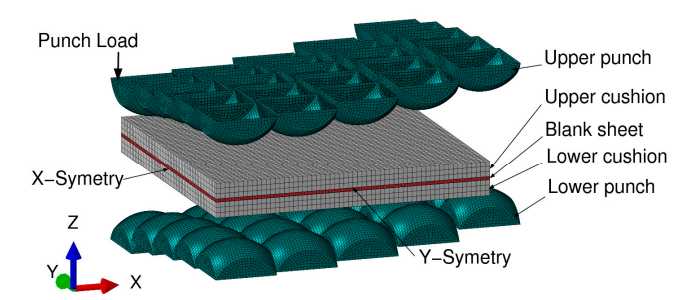

Figure 2. Finite element model for saddle shape.

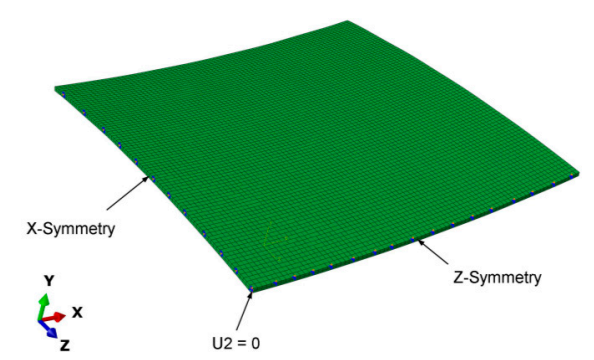

Figure 3. Simulation result before springback and the setup for implicit analysis.

\subsection{Defect Quantification}

The main objective of the study is investigating the process parameters to compensate for the springback for the given target shape. The study also tried to reduce the induced springback for a different blank thickness. As shown in Figure 4, a numerical simulation was conducted with the process parameters of target curvature radius $800 \mathrm{~mm}$, blank cross-section $320 \mathrm{~mm} \times 320 \mathrm{~mm}$, blank thickness $2 \mathrm{~mm}$, punch width $40 \mathrm{~mm}$, punch-tip radius $30 \mathrm{~mm}$, elastic cushion thickness $16 \mathrm{~mm}$, punch displacement (elastic cushion compressive strain ratio) $6 \%$, and the punch holding time is $0 \mathrm{~s}$. As shown in the figure, the springback is observed from nodal displacement distribution before and after unloading of the punch load. The study used the maximum deviation of the vertical displacement $U 2$ from the expected target shape to quantify the springback responses of the numerical simulation result. The equation is given as:

$$
f(u)=\max _{1<i<N}\left(\left|u_{\mathrm{t}}^{i}-u_{\mathrm{d}}^{i}\right|\right)
$$

where $N$ is the number of nodes, $u_{\mathrm{t}}^{i}$ and $u_{\mathrm{d}}^{i}$ is the nodal displacement at the $i$ th node of the target and deformed shape, respectively. 


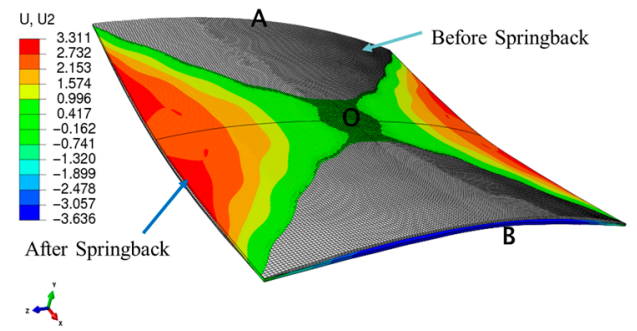

(a)

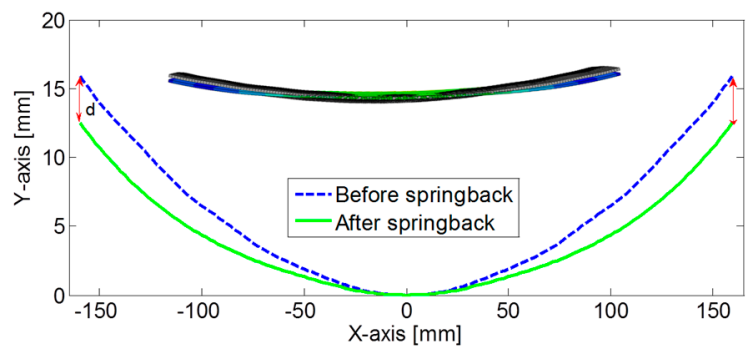

(b)

Figure 4. Case of visible springback: (a) Nodal displacement distribution; (b) Curvature radii at section AB.

\section{Surrogate Modeling}

\subsection{Process Parameter}

As mentioned in the previous section, the study has two objectives, the first one is reducing the springback by setting the process parameters and the second one is compensating the induced springback for the expected target shape. Springback is induced because of the elastic recovery (deformation) after the forming process is performed. Previous researchers suggested that creating a restraining force, such as using a blank holder, can decrease the amount of the springback by increasing the sheet tension; however, in our case, there is no blank holder to create such kinds of tension load during the forming process. Here, the study suggested that holding the upper punch load for a certain time can stabilize the induced stress. To check the sensitivity of the process in regards to the punch holding time, the study run a numerical simulations in ABAQUS by varying the punch holding time of $0,2.5,5,10,20,30,40,50$, and $60 \mathrm{~s}$. The other parameters were the same as in Section 2.4. During the quasi-static simulation in ABAQUS, stopping the punch means stopping the simulation, so, to investigate the punch holding time, the study applied a macro speed for the specified time at the end of the forming process and considering it as a punch holding time. As Figure 5 shows, in the first few seconds, the springback result is drastically decreased, however, after that, the springback responses became the same for a few seconds, and getting a slightly increased while the punch holding time is increased. So, to find the optimal punch hold time, the study investigates in between 0 and $20 \mathrm{~s}$.

To compensate the induced springback value, the only option in MDF is changing the curvature radius. Here, the study target curvature radius is $800 \mathrm{~mm}$. To check the upper and lower value, the study conducted two FE simulations. As shown in Figure 6, the study target curvature is between the simulation result of 600 and $800 \mathrm{~mm}$ curvature radii after springback. So, the study searches the optimal radius value in between 600 and $800 \mathrm{~mm}$ curvature radius for different blank thickness. The considered blank thickness in this study is in between 0.5 and $2 \mathrm{~mm}$. The process parameters are summarized in Table 2.

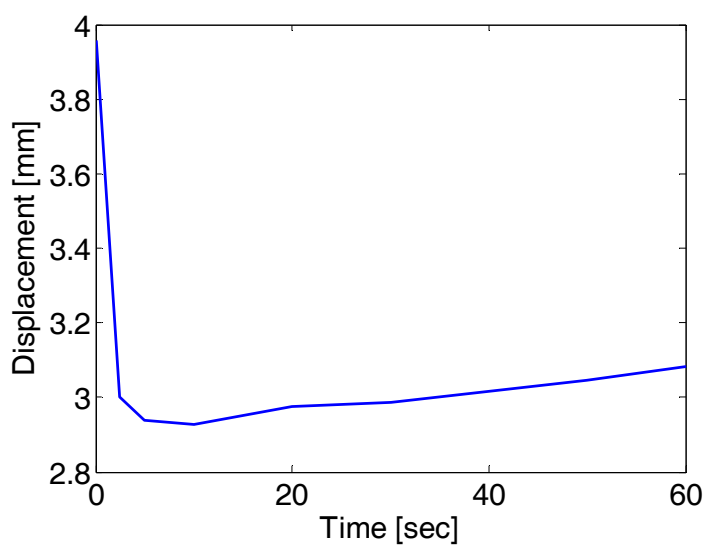

Figure 5. Punch holding time effect on springback. 


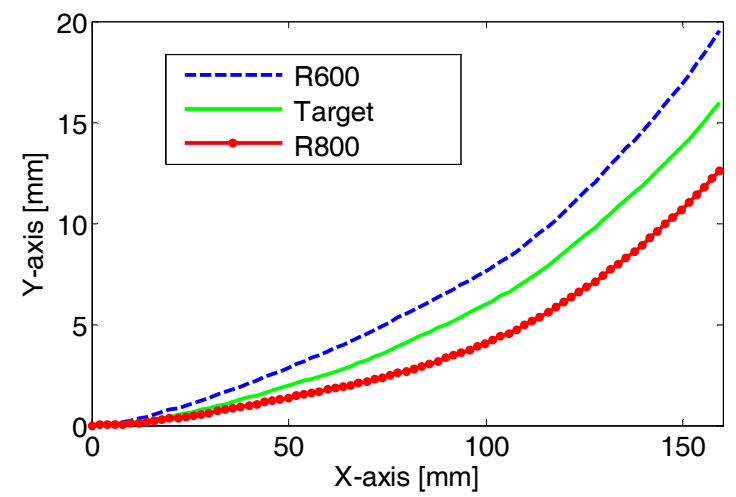

Figure 6. Target curvature radius with the deformed result of $R=600 \mathrm{~mm}$ and $R=800 \mathrm{~mm}$ curvature radius after springback.s

Table 2. Process parameters.

\begin{tabular}{cccc}
\hline Parameters & Lower Bound & Upper Bound & Units \\
\hline Curvature radius & 600 & 800 & $\mathrm{~mm}$ \\
Blank thickness & 0.5 & 2 & $\mathrm{~mm}$ \\
Blank holding time & 0 & 20 & $\mathrm{~s}$ \\
\hline
\end{tabular}

\subsection{Sample Stratagey}

To develop the prediction model, the study generates 30 sample data for training using Latin hypercube sampling method. Latin hypercube sampling is one of the best DOE techniques which efficiently sample the large design spaces by maximizing the minimum distance between all of the possible pairs of the sample points in a given sample plan, this shows that a Latin hypercube design method will keep a distance between the samples as large as possible. Figure 7a shows the scatter plots of the training sample data and the views of the plot also shows that the sample is taken only one sample from each row and each column. In addition, 30 random sample data were generated using the MATLAB built function for testing of the developed prediction model. Figure $7 \mathrm{~b}$ shows the scatter plots of the testing sample data, as compared to the training data, the random data may have a chance to take a sample from the same row and column, which is shown in the front view of the figure. Tables A1 and A2 also show the training and testing raw data, respectively.
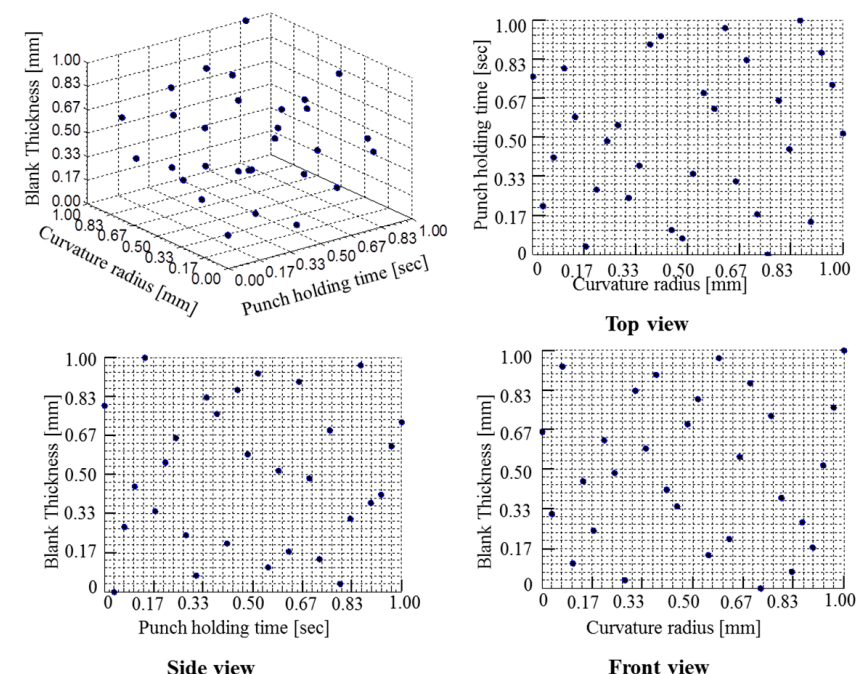

(a)

Figure 7. Cont. 

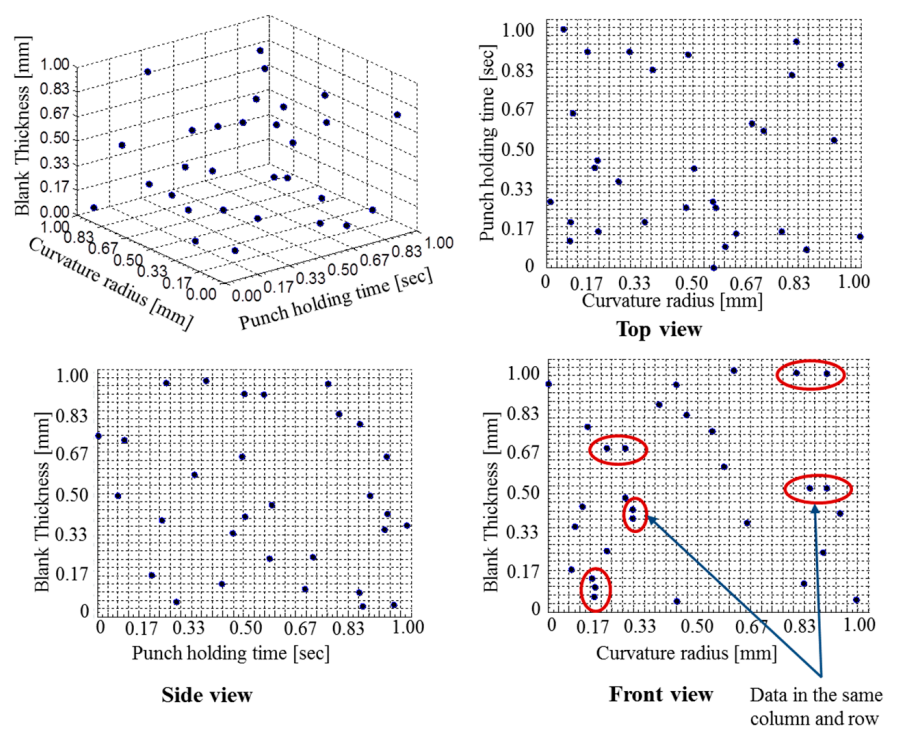

(b)

Figure 7. Training and testing sample data of the model: (a) Training data using Latin hypercube sampling; and (b) Testing data using random sampling.

\subsection{Radial Basis's Function Formulation}

RBF was first introduced by Rolland Hardy to fit irregular topographic contours of geographical data [20]. RBF networks are three layer feed-forward networks that are trained using a supervised training algorithm, which is shown in Figure 8, featuring are an input $x$, hidden units $\Psi$, weights $w$, linear output transfer functions, and output $f(x)$. To construct the fitting model using RBF, we first consider the scalar response function $f$ or the yielding responses $y=\left\{y_{1}, y_{2}, \ldots, y_{n}\right\}^{\mathrm{T}}$ which is obtained from the simulation or experimental results by employing the input training sample data of $X=\left\{x_{1}, x_{2}, \ldots, x_{n}\right\}^{\mathrm{T}}$. Then, we pursue RBF approximation function $\hat{f}(\boldsymbol{x})$, which is given as

$$
\hat{f}(\boldsymbol{x})=w^{\mathrm{T}} \mathbf{\Psi}=\sum_{i=1}^{n_{\mathrm{c}}} w_{i} \Psi_{i}\left(\left\|\boldsymbol{x}-\boldsymbol{c}_{i}\right\|\right)
$$

where $\mathbf{c}_{i}$ is the $i$ th of the $n_{\mathrm{c}}$ basis function centers and $\boldsymbol{\Psi}$ denotes the $n_{\mathrm{c}}$ vector which is contained the values of the basis function $\Psi$, it is evaluated at the Euclidean distances between the prediction site $x$ and the centers $c_{i}$ of the basis functions, $w_{i}$ denotes the weight of the $i$ th basis function. In a previous study, different basis functions are suggested, which is used in RBF, some of the basis functions are stated as follow under categories of fixed and parametric basis functions:

1. Fixed basis functions

- Linear: $\Psi(x)=\left(x-c_{i}\right)$

- Cubic: $\Psi(x)=\left(x-c_{i}\right)^{3}$

- Thin plate spline: $\Psi(x)=\left(x-c_{i}\right)^{2} \ln \left(x-c_{i}\right)$

2. Parametric basis functions

- Gaussian: $\Psi(x)=\mathrm{e}^{-\left(x-c_{i}\right)^{2} /\left(2 \sigma^{2}\right)}$

- Multiquadratic: $\Psi(x)=\left(\left(x-c_{i}\right)^{2}+\sigma^{2}\right)^{1 / 2}$

- Inverse multiquadratic: $\Psi(x)=\left(\left(x-c_{i}\right)^{2}+\sigma^{2}\right)^{-1 / 2}$ 


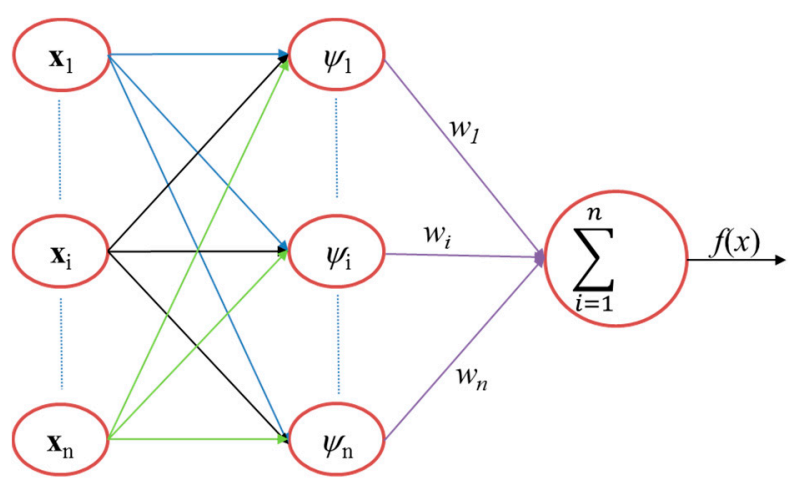

Figure 8. Radial basis function network.

As we have seen from the formulation equation, the weight $w$ parameters are unknown, in addition, for parametric basis functions, the variance (width) $\sigma$ is also unknown. The estimation of the weight parameters can be found through the interpolation of

$$
y\left(x_{j}\right)=\sum_{i=1}^{n_{\mathrm{c}}} w_{i} \Psi_{i}\left(\left\|\boldsymbol{x}-\boldsymbol{c}_{i}\right\|\right), j=1,2, \cdots, n .
$$

The above equation is linear in terms of the weights $w$ of the basis function; however, the predictor $y$ can express highly nonlinear. To obtain a unique solution, the system in Equation (6) has to be square; this means that $n_{\mathrm{c}}$ should be equal to $n$. For simplification, the bases coincide with the data points, which means $c_{i}=x_{i}$, for $i=1,2, \ldots, n$, this leads us to the equation of

$$
\Psi w=y,
$$

where $\boldsymbol{\Psi}$ is also called as Gram matrix and it is defined as $\Psi=\Psi\left(\left\|\boldsymbol{x}_{j}-\boldsymbol{x}_{i}\right\|\right), i=j=1,2, \ldots, n$. From Equation (7) we can estimate the weight value as

$$
w=\Psi^{-1} \boldsymbol{y}
$$

However, if the responses $y=\left\{y_{1}, y_{2}, \ldots, y_{n}\right\}^{\mathrm{T}}$ are corrupted by noise, using the above equation may affect the prediction model that to fit the observed data. The noise effect should be considered in the model. To solve this kind of problem, Poggio and Girosi [21] introduced using a regularization parameter $\lambda$ as model flexibility can control the noise effect on the prediction model. It is also recommended that $\lambda$ value should be adequately small, such as $\lambda=1.0 \times 10^{-3}$. This will add to the diagonal matrix of $\boldsymbol{\Psi}$. So the weight estimation value will be in the form of

$$
w=\left(\boldsymbol{\Psi}^{\mathrm{T}} \boldsymbol{\Psi}+\lambda \boldsymbol{I}\right) \mathbf{\Psi}^{\mathrm{T}} \boldsymbol{y},
$$

where $\Psi$, and $\lambda \boldsymbol{I}$ are given as follows:

$$
w=\left[\begin{array}{cccc}
\Psi_{1}\left(x_{1}\right) & \Psi_{2}\left(x_{1}\right) & \ldots & \Psi_{n}\left(x_{1}\right) \\
\Psi_{1}\left(x_{2}\right) & \Psi_{2}\left(x_{2}\right) & \ldots & \Psi_{n}\left(x_{2}\right) \\
\vdots & \vdots & \ddots & \vdots \\
\Psi_{1}\left(x_{n}\right) & \Psi_{2}\left(x_{n}\right) & \ldots & \Psi_{n}\left(x_{n}\right)
\end{array}\right]
$$




$$
\boldsymbol{I}=\left[\begin{array}{cccc}
1 & 0 & \cdots & 0 \\
0 & 1 & \cdots & 0 \\
\vdots & \vdots & \ddots & \vdots \\
0 & 0 & \cdots & 1
\end{array}\right]
$$

To estimate the sigma value the previous studies proposed different methods, for instance, Nakayama, et al. [22] proposed $\sigma=\frac{d_{\max }}{\sqrt[m]{n m}}$, where $n$ denotes the number of sampling point, $m$ denotes the number of process parameters, and $d_{\max }$ is denotes the maximum distance among the sampling point. Whereas, the accurate estimation of the sigma parameters will allow for us to reduce the generalization (estimated) error of the prediction model, so, this study used the direct search method by investigating the model accuracy using the cross-validation error for each sigma value. Cross-validation is a model verification method, which is used to assess the results of a statistical analysis to simplify the independent data set. Here, the study investigates the model by removing some data from the training sample data and evaluating each sigma value for every removed data and finally we chosen the best sigma value, which has a minimum model cross-validation error. The cross-validation function is given as:

$$
E_{\mathrm{crv}}=\frac{1}{q} \sum_{i=1}^{q}\left[y\left(x_{i}\right)-\hat{f}\left(x_{i}, w\right)\right],
$$

where $q$ is the number of the removed data from the training sample (subsets of the training sample), $y\left(x_{i}\right)$, and $\hat{f}\left(x_{i}, w\right)$ is the true and prediction response value at the $i$ th removed training sample, respectively. If $q$ is equal to the number of training sample data $n$, the cross-validation error is a nearly balanced estimator of the exact risk. Nevertheless, because of the $n$ subsets being similar to each other the leave-one-out measure variance can be quite high. Hastie et al. [23] proposed a desirable value of $q$ such as $q=5$ or $q=10$, it is depends on the total number of sample data. In general, using less number of the training sample subsets $q$ means reducing the cross-validation process computational cost by reducing the total number of the fitted model.

Here, the study used 30 numbers of samples, so we chose $q$ is equal to 5 and the $\sigma$ value is searched in between $10^{-2}$ and $10^{2}$. The algorithm is written in MATLAB and the $\sigma$ value found as 1.6103. In addition, the obtained weight $w$ value also listed in Table 3.

Table 3. The weight value.

\begin{tabular}{cc}
\hline No. & Value \\
\hline 1 & -0.1325 \\
2 & 2.7338 \\
3 & 0.849 \\
4 & -0.3378 \\
5 & -0.0510 \\
6 & -2.4592 \\
7 & 0.9597 \\
8 & -1.9367 \\
9 & -0.5711 \\
10 & -2.6565 \\
11 & -0.6119 \\
12 & 1.2294 \\
13 & 3.2205 \\
14 & 1.7304 \\
15 & -1.4591 \\
16 & -1.0661 \\
17 & -2.6649 \\
18 & -3.1082 \\
19 & 7.7967 \\
\hline
\end{tabular}


Table 3. Cont.

\begin{tabular}{cc}
\hline No. & Value \\
\hline 20 & -2.9087 \\
21 & 1.2768 \\
22 & 0.6473 \\
23 & 3.0969 \\
24 & -0.0234 \\
25 & -2.3770 \\
26 & -1.7703 \\
27 & 0.3146 \\
28 & 0.2355 \\
29 & -0.1235 \\
30 & 0.4802 \\
\hline
\end{tabular}

\subsection{Numerical Model Verification}

For prediction model verification, the roots mean square error (RMSE), and co-efficient of determination $\left(R^{2}\right)$ numerical verification techniques are used in this study. Table 4 compared the model verification of different prediction methods, as we have seen from the model verification results, RBF with multiquadratic basis function have better prediction than the others. Figure 9 shows of the predicted vs. the observed result comparison for RBF with a multiquadratic basis function; the predicted and observed result is quietly fitted to each other. Figure 10 also shows that the residual values are randomly distributed and the residual mean value also almost zero, this verified that the developed model is acceptable to use.

Table 4. Results of model verification for different prediction models.

\begin{tabular}{cccc}
\hline Model & $\boldsymbol{R}^{\mathbf{2}}$ & RMSE \\
\hline Blind Kriging 2nd deg. & 0.9837 & 0.0518 \\
Blind Kriging 3rd deg. & 0.9848 & 0.0501 \\
Ordinary Kriging & 0.9589 & 0.4510 \\
\hline Linear & 0.9811 & 0.0500 \\
Cubic & 0.9854 & 0.0444 \\
RBF & Thin plate spline & 0.8728 & 0.1297 \\
& Gaussian & 0.9868 & 0.0418 \\
& Multiquadratic & 0.9935 & 0.0294 \\
& Inverse multiquadratic & 0.9717 & 0.0612 \\
\hline
\end{tabular}

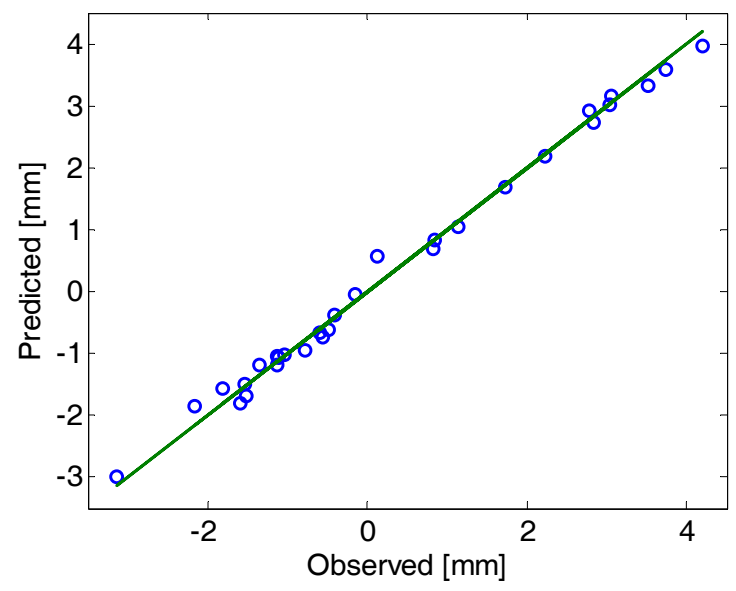

Figure 9. Predicted vs. observed result for RBF with multiquadratic basis function. 


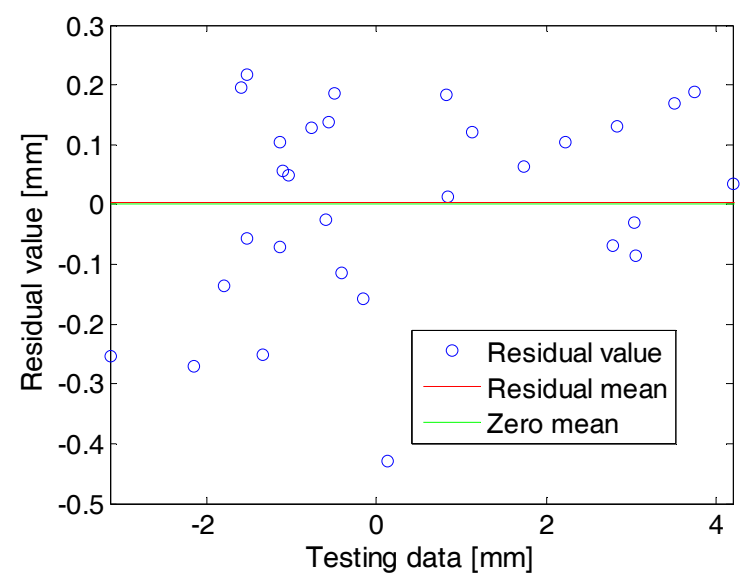

Figure 10. Residual error.

\section{Process Optimization}

\subsection{Optimization Formulation}

The objectives of the study are reducing the springback and compensating the induced springback for the expected target shape. As explored in Section 2.4, the springback is examined using the maximum deviation of the vertical displacement of the deformed shape from the expected target shape for both objectives. The formulation function is given as

$$
\begin{aligned}
& \min f(x) \\
& \text { s.t. } x^{l} \leq x_{i} \leq x^{u}
\end{aligned}
$$

where $x\left(x_{1}, x_{2}\right.$, and $\left.x_{3}\right)$ is the process parameters, here, the constructed function is the same for both of the objectives. The first objective is to minimize the induced springback for a given curvature radius and blank thickness. So, the curvature radius and blank thickness should be fixed, the only parameter left here is the punch holding time before unloading of the punch load. The second objective is minimizing the maximum curvature radius difference from the target shape to compensate for the expected shape error due to springback. In this case, the punch holding time is fixed to the obtained optimal value of the first objective for the given target curvature radius and blank thickness.

\subsection{Result and Discussion}

After developing the prediction model using RBF as a function of curvature radius, punch holding time, and blank thickness, the study employed genetic algorithm on MATLAB to determine the global optimal value of the given objective. The algorithm scheme is shown in Figure 11. From the first objective, the optimal value of punch holding time is obtained to reduce the springback for the fixed curvature radius and blank thickness. After obtaining the proper punch holding time, we proceed to find the optimal value of the curvature radius to compensate for the induced springback for the given target shapes, in our case, the target curvature radius was $800 \mathrm{~mm}$ and the blank thickness was $2 \mathrm{~mm}$. Table 5 summarizes the obtained process parameter setting to compensate for the induced springback for the expected target shape. Figure 12 shows the curvature radii comparison between the simulation result and the target shape. The RMSE between the simulation result and target shape is 0.0245 , this shows us the obtained result is acceptable with the mentioned error. 
Table 5. Optimal result.

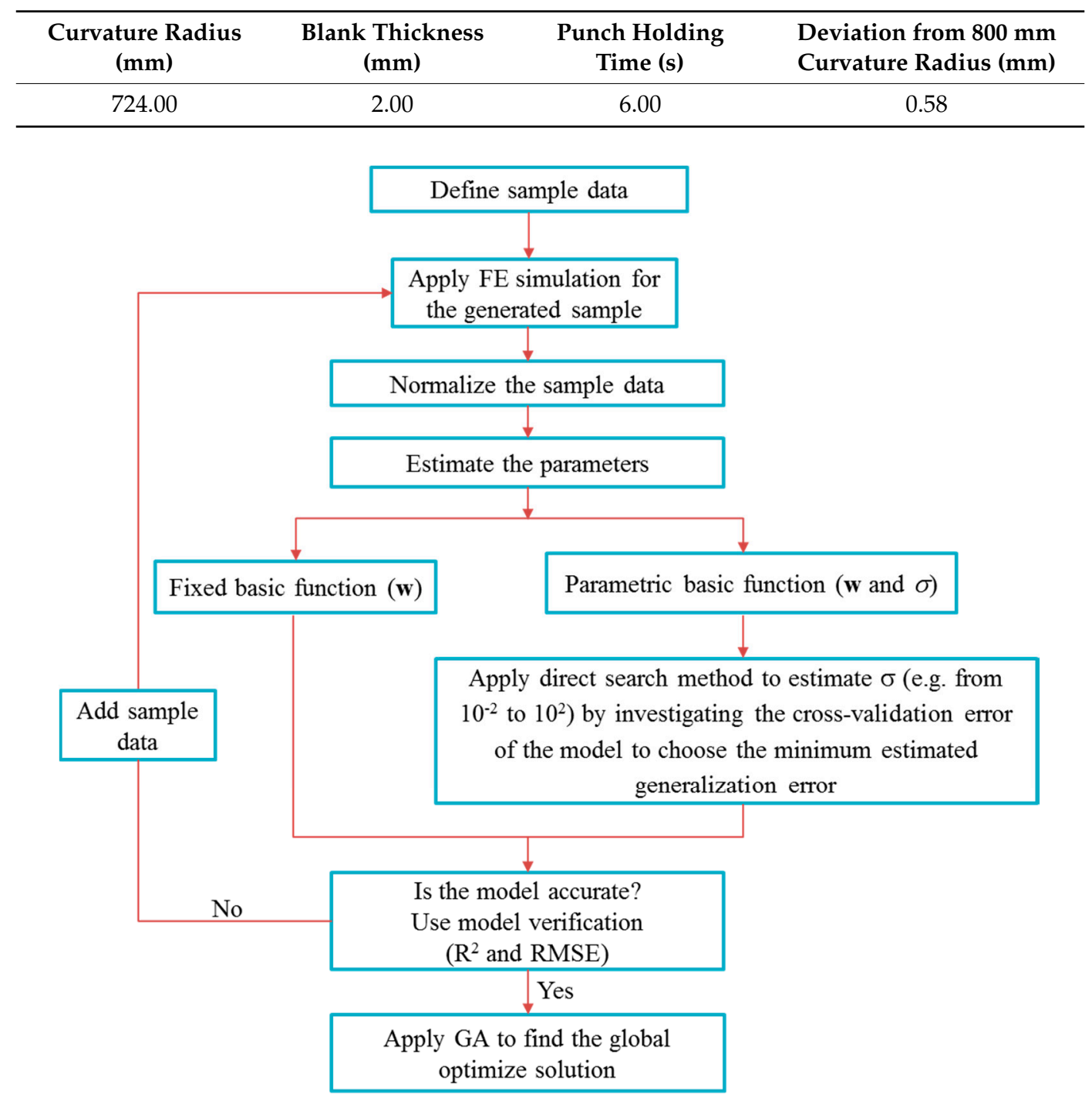

Figure 11. Radial basis optimization algorithm.

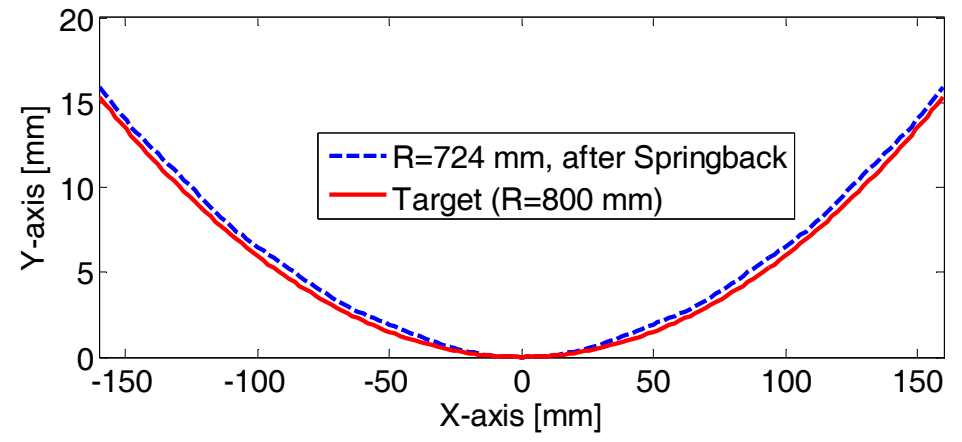

Figure 12. Compensated optimal result.

\section{Conclusions}

The induced springback in MDF process is investigated using ABAQUS commercial software, and the study tried to reduce the springback by finding the optimal punch holding time. The study also compensates for the induced springback by finding the optimal curvature radius, using the developed 
surrogate model for different blank thickness. The prediction model was chosen after investigating different types of the prediction model using the model verification of $R^{2}$ and RMSE. The study chose RBF with a multi-quadratic base function to develop the model as a function of punch holding time, curvature radius, and blank thickness. Finally, the study found the optimal curvature radius, and the punch holding time for the target curvature and blank thickness with the RMSE of 0.0245 by employing the GA on MATLAB.

Acknowledgments: This work was supported by the National Research Foundation of Korea (NRF) grant funded by the Korea government (MSIP) through the Engineering Research Center (No. 2012R1A5A1048294). Also, this work was supported by the Human Resource Training Program for the Regional Innovation and Creativity through the Ministry of Education and National Research Foundation of Korea (NRF-2015H1C1A1035499).

Author Contributions: Misganaw Abebe and Jun-Seok Yoon conceived and designed the experimental data; Misganaw Abebe and Beom-Soo Kang analyzed the data; Misganaw Abebe wrote the algorithm on MATLAB, Misganaw Abebe and Jun-Seok Yoon wrote the paper and edited by Beom-Soo Kang.

Conflicts of Interest: The authors declare no conflict interest.

\section{Appendix A}

Table A1. Training sample data.

\begin{tabular}{|c|c|c|c|}
\hline Case & Punch Holding Time, $x_{1}(\mathrm{~s})$ & Curvature Radius, $x_{2}(\mathrm{~mm})$ & Thickness, $x_{3}(\mathrm{~mm})$ \\
\hline 1 & 14.482 & 766 & 0.862 \\
\hline 2 & 13.104 & 738 & 1.741 \\
\hline 3 & 8.966 & 779 & 0.655 \\
\hline 4 & 17.932 & 772 & 1.172 \\
\hline 5 & 10.344 & 731 & 1.379 \\
\hline 6 & 2.068 & 641 & 1.069 \\
\hline 7 & 4.138 & 745 & 0.552 \\
\hline 8 & 18.620 & 628 & 0.914 \\
\hline 9 & 11.034 & 662 & 1.793 \\
\hline 10 & 8.276 & 614 & 1.276 \\
\hline 11 & 4.828 & 703 & 1.535 \\
\hline 12 & 2.758 & 683 & 1.948 \\
\hline 13 & 15.172 & 800 & 1.483 \\
\hline 14 & 13.794 & 634 & 0.604 \\
\hline 15 & 16.552 & 710 & 1.017 \\
\hline 16 & 20.000 & 697 & 1.690 \\
\hline 17 & 17.242 & 600 & 2.000 \\
\hline 18 & 12.414 & 607 & 1.638 \\
\hline 19 & 9.656 & 786 & 1.897 \\
\hline 20 & 0.000 & 648 & 1.586 \\
\hline 21 & 19.31 & 655 & 0.500 \\
\hline 22 & 0.690 & 759 & 1.431 \\
\hline 23 & 6.896 & 724 & 1.845 \\
\hline 24 & 11.724 & 676 & 0.810 \\
\hline 25 & 15.862 & 669 & 1.328 \\
\hline 26 & 1.380 & 717 & 1.121 \\
\hline 27 & 5.518 & 690 & 0.707 \\
\hline 28 & 3.448 & 793 & 0.965 \\
\hline 29 & 6.206 & 752 & 1.224 \\
\hline 30 & 7.586 & 621 & 0.759 \\
\hline
\end{tabular}


Table A2. Testing sample data.

\begin{tabular}{|c|c|c|c|}
\hline Case & Punch Holding Time, $x_{1}(\mathrm{~s})$ & Curvature Radius, $x_{2}(\mathrm{~mm})$ & Thickness, $x_{3}(\mathrm{~mm})$ \\
\hline 1 & 1.518 & 779 & 1.126 \\
\hline 2 & 1.080 & 608 & 0.575 \\
\hline 3 & 10.616 & 800 & 1.854 \\
\hline 4 & 15.584 & 645 & 1.917 \\
\hline 5 & 18.680 & 637 & 1.236 \\
\hline 6 & 2.598 & 626 & 1.234 \\
\hline 7 & 11.376 & 783 & 1.007 \\
\hline 8 & 9.388 & 720 & 1.850 \\
\hline 9 & 0.238 & 747 & 1.054 \\
\hline 10 & 6.742 & 641 & 0.667 \\
\hline 11 & 3.244 & 714 & 1.670 \\
\hline 12 & 15.886 & 618 & 1.085 \\
\hline 13 & 6.224 & 763 & 0.863 \\
\hline 14 & 10.570 & 747 & 1.106 \\
\hline 15 & 3.312 & 771 & 0.645 \\
\hline 16 & 12.040 & 773 & 0.698 \\
\hline 17 & 5.260 & 626 & 1.913 \\
\hline 18 & 13.082 & 684 & 1.934 \\
\hline 19 & 13.784 & 690 & 1.363 \\
\hline 20 & 14.964 & 771 & 0.590 \\
\hline 21 & 9.010 & 628 & 0.852 \\
\hline 22 & 1.676 & 676 & 1.030 \\
\hline 23 & 4.580 & 731 & 1.732 \\
\hline 24 & 18.266 & 697 & 1.573 \\
\hline 25 & 3.048 & 720 & 0.565 \\
\hline 26 & 16.516 & 786 & 0.754 \\
\hline 27 & 10.766 & 752 & 1.474 \\
\hline 28 & 19.922 & 775 & 1.598 \\
\hline 29 & 1.564 & 763 & 1.472 \\
\hline 30 & 8.854 & 752 & 1.176 \\
\hline
\end{tabular}

\section{References}

1. Li, L.; Seo, Y.H.; Heo, S.C.; Kang, B.S.; Kim, J. Numerical simulations on reducing the unloading springback with multi-step multi-point forming technology. Int. J. Adv. Manuf. Technol. 2010, 48, 45-61. [CrossRef]

2. Hwang, S.Y.; Lee, J.H.; Yang, Y.S.; Yoo, M.J. Springback adjustment for multi-point forming of thick plates in shipbuilding. Comput. Aided Des. 2010, 42, 1001-1012. [CrossRef]

3. Shim, D.S.; Baek, G.Y.; Shin, G.Y.; Yoon, H.S.; Lee, K.Y.; Kim, K.H. Investigation of tension force in stretch forming of doubly curved aluminum alloy (A15083) sheet. Int. J. Precis. Eng. Manuf. 2016, 17, 433-444. [CrossRef]

4. Liu, W.; Liang, Z. Springback compensation for multi-curvature part based on multi-objective optimization of fuzzy genetic algorithm. In Proceedings of the IEEE Chinese Control and Decision Conference, Guilin, China, 17-19 June 2009; pp. 3659-3664.

5. Zhang, Q.F.; Cai, Z.Y.; Zhang, Y.; Li, M.Z. Springback compensation method for doubly curved plate in multi-point forming. Mater. Des. 2013, 47, 377-385. [CrossRef]

6. Kitayama, S.; Yoshioka, H. Springback reduction with control of punch speed and blank holder force via sequential approximate optimization with radial basis function network. Int. J. Mech. Mater. Des. 2014, 10, 109-119. [CrossRef]

7. Behera, A.K.; Verbert, J.; Lauwers, B.; Duflou, J.R. Tool path compensation strategies for single point incremental sheet forming using multivariate adaptive regression splines. Comput. Aided Des. 2013, 45, 575-590. [CrossRef]

8. Li, E. Reduction of springback by intelligent sampling-based LSSVR metamodel-based optimization. Int. J. Mater. Form. 2013, 6, 103-114. [CrossRef] 
9. Khadra, F.A.; El-Morsy, A.W. Prediction of Springback in the Air Bending Process Using a Kriging Metamodel. Eng. Technol. Appl. Sci. Res. 2016, 6, 1200.

10. ul Hassan, H.; Fruth, J.; Güner, A.; Mennecart, T.; Tekkaya, A.E. Finite element simulations for sheet metal forming process with functional input for the minimization of springback. In Proceedings of the IDDRG Conference, Zurich, Switzerland, 2-5 June 2013; pp. 393-398.

11. Karaağaç, İ. The evaluation of process parameters on springback in V-bending using the flexforming process. Mater. Res. 2017, 20, 1291-1299. [CrossRef]

12. Tekaslan, Ö.; Gerger, N.; Şeker, U. Determination of spring-back of stainless steel sheet metal in " $\mathrm{V}^{\text {" bending }}$ dies. Mater. Des. 2008, 29, 1043-1050. [CrossRef]

13. Choudhury, I.A.; Ghomi, V. Springback reduction of aluminum sheet in V-bending dies. Proc. Inst. Mech. Eng. Part B 2014, 228, 917-926. [CrossRef]

14. Jakumeit, J.; Herdy, M.; Nitsche, M. Parameter optimization of the sheet metal forming process using an iterative parallel Kriging algorithm. Struct. Multidiscip. Optim. 2005, 29, 498-507. [CrossRef]

15. Naceur, H.; Guo, Y.Q.; Ben-Elechi, S. Response surface methodology for design of sheet forming parameters to control springback effects. Comput. Struct. 2006, 84, 1651-1663. [CrossRef]

16. Wei, L.; Yang, Y.; Xing, Z.; Zhao, L. Springback control of sheet metal forming based on the response-surface method and multi-objective genetic algorithm. Mater. Sci. Eng. A 2009, 499, 325-328. [CrossRef]

17. Abebe, M.; Lee, K.; Kang, B.S. Surrogate-based multi-point forming process optimization for dimpling and wrinkling reduction. Int. J. Adv. Manuf. Technol. 2016, 85, 391-403. [CrossRef]

18. Heo, S.C.; Seo, Y.H.; Yoon, J.S.; Song, W.J.; Kang, B.S. Effect of design variables on forming accuracy in thick plate flexible forming process. In Proceedings of the 14th International conference on Metal Forming, AGH University of Science and Technology, Krakow, Poland, 16-19 September 2012; pp. 1403-1406.

19. Abebe, M.; Park, J.W.; Kang, B.S. Reliability-based robust process optimization of multi-point dieless forming for product defect reduction. Int. J. Adv. Manuf. Technol. 2017, 89, 1223-1234. [CrossRef]

20. Hardy, R.L. Multiquadric equations of topography and other irregular surfaces. J. Geophys. Res. 1971, 76, 1905-1915. [CrossRef]

21. Poggio, T.; Girosi, F. Regularization algorithms for learning that are equivalent to multilayer networks. Science 1990, 247, 978-982. [CrossRef] [PubMed]

22. Nakayama, H.; Masao, A.; Rie, S. Simulation-based optimization using computational intelligence. Optim. Eng. 2002, 3, 201-214. [CrossRef]

23. Hastie, T.; Tibshirani, R.; Friedman, J. The Elements of Statistical Learning; Springer: New York, NY, USA, 2001. 